\title{
Diffusion Tensor Tractography for Determining Injury to the Oculomotor Nerve in a Patient With Cerebral Infarct
}

\author{
Sungho Jang, MD, Soyoung Kwak, MD, MPH, PhD
}

Department of Physical Medicine and Rehabilitation, Yeungnam University College of Medicine, Daegu, Korea

A 39-year-old male patient, diagnosed with a cerebral infarct in the left midbrain, visited Yeungnam University Hospital and underwent conservative management. Brain magnetic resonance imaging performed at 1 week from onset showed a leukomalactic lesion in the left midbrain (Fig. 1A). Mild ptosis ( $3 \mathrm{~mm}$ ) and mild limitation in the upward $\left(20^{\circ}\right)$ and medical $\left(10^{\circ}\right)$ gaze were observed. However, his left eye was not deviated, and did not show mydriasis.

Diffusion tensor imaging (DTI) data were acquired one week after onset. A 6-channel head coil on a 1.5T Philips Gyroscan Intera (Philips, Best, The Netherlands) with single-shot echo-planar imaging was used for scanning. DTI-Studio software was used for the reconstruction of the oculomotor nerve. Fibers passing through two regions of interest (ROIs) were depicted with a fractional anisotropy of $>0.2$ and a tract turning-angle of $<60^{\circ}$. Following the known anatomy, the first ROI was given at the periaqueductal region, and the second one at the oculomotor nerve area of the ventral part of the midbrain, with an option of cut operation on the axial images [1-5].

Diffusion tensor tractography (DTT) revealed a discontinuation of the left oculomotor nerve in the area where the nerve exited the left midbrain (Fig. 1B). This patient showed mild ptosis and limitations of eye movement in his left eye; these mild dysfunctions appeared to result from the injured left oculomotor nerve, caused by the left midbrain infarct. Our results suggest that DTT is useful to demonstrate injury to the oculomotor nerve for patients with clinical features of oculomotor nerve palsy after cerebral infarct [1-5].

\section{CONFLICT OF INTEREST}

No potential conflict of interest relevant to this article was reported.

\section{ACKNOWLEDGMENTS}

This work was supported by the National Research Foundation (NRF) of Korea grant funded by the Korean Government (MSIP) (No. 2015R1A2A2A01004073).

\section{REFERENCES}

1. Yamada K, Shiga K, Kizu O, Ito H, Akiyama K, Nakagawa $\mathrm{M}$, et al. Oculomotor nerve palsy evaluated by diffusion-tensor tractography. Neuroradiology 2006; 

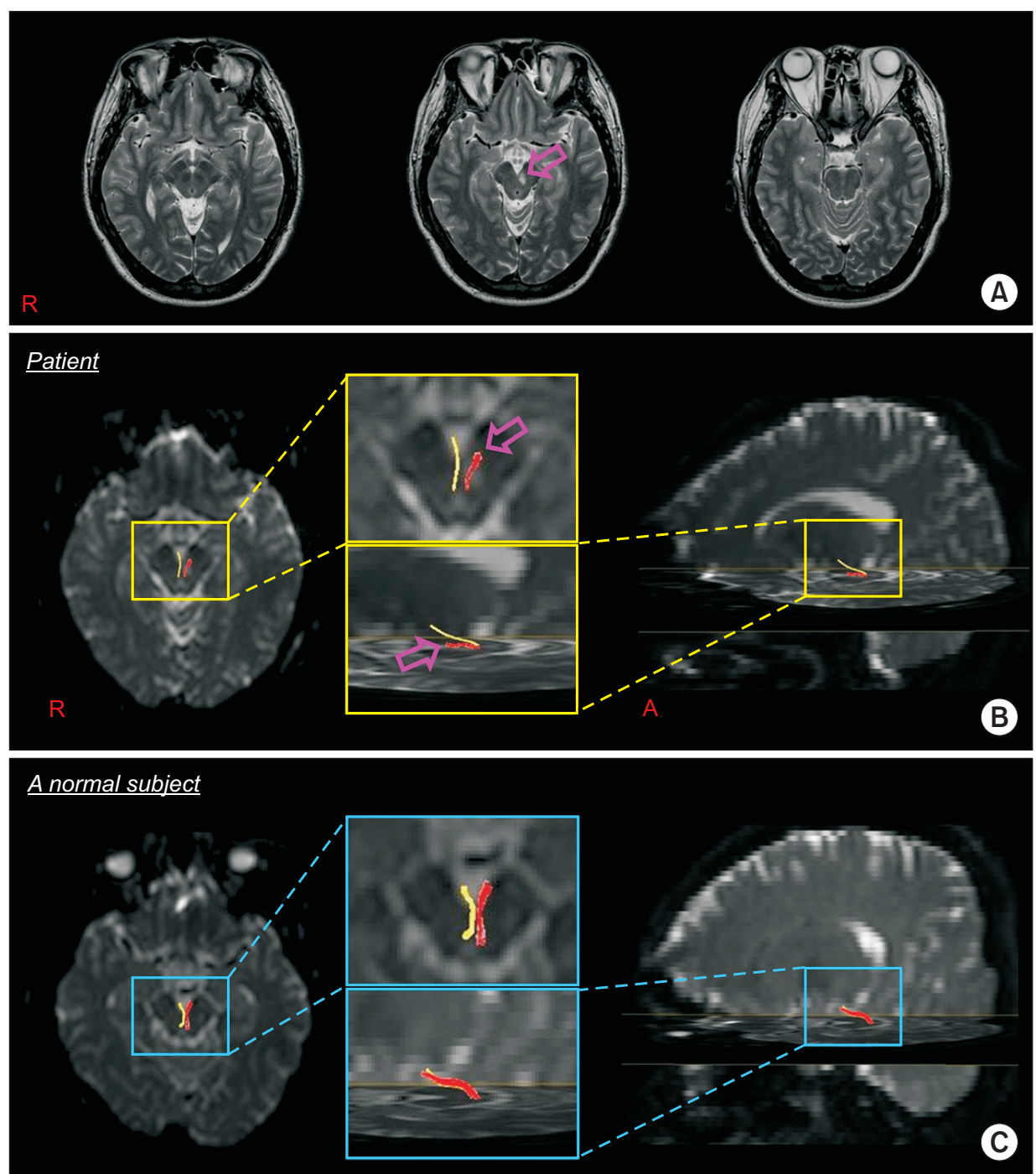

Fig. 1. (A) T2-weighted brain magnetic resonance images at 1 week from onset, reveals leukomalactic lesion in the left midbrain. (B, C) Diffusion tensor tractography of the left oculomotor nerve shows a discontinuation (arrow) in the area where the left nerve exits the left midbrain, as compared to the right oculomotor nerve of the patient and a normal control subject (40-year-old male).
48:434-7.

2. Hodaie M, Quan J, Chen DQ. In vivo visualization of cranial nerve pathways in humans using diffusionbased tractography. Neurosurgery 2010;66:788-95; discussion 795-6.

3. Seo YS, Chang CH, Jung YJ, Jang SH. Injury of the oculomotor nerve after aneurysmal subarachnoid hemorrhage: diffusion tensor tractography study. Am J Phys Med Rehabil 2015;94:e51-2.
4. Kwon HG, Jang SH. Recovery of injured oculomotor nerve in a patient with intracerebral hemorrhage. Am J Phys Med Rehabil 2014;93:1107-8.

5. Kwon HG, Kim MS, Kim SH, Jang SH. Neurological picture. Injury of the oculomotor nerve in a patient with traumatic brain injury: diffusion tensor tractography study. J Neurol Neurosurg Psychiatry 2013;84: 1073-4. 